\title{
Immunohistochemical analysis of phosphorylated mammalian target of rapamycin and its downstream signaling components in invasive breast cancer
}

\author{
BIN-LIN MA ${ }^{1}$, MEI-HUI SHAN ${ }^{1}$, GANG SUN ${ }^{1}$, GUANG-HUI REN ${ }^{1}$,

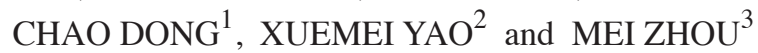 \\ ${ }^{1}$ Department of Breast and Neck, The Affiliated Tumor Hospital of Xinjiang Medical University; \\ ${ }^{2}$ Department of Epidemiology and Health Statistics, Public Health College of Xinjiang Medical University; \\ ${ }^{3}$ Department of Pathology, The Affiliated Tumor Hospital of Xinjiang Medical University, \\ Urumqi, Xinjiang 830011, P.R. China
}

Received August 18, 2014; Accepted April 30, 2015

DOI: $10.3892 / \mathrm{mmr} .2015 .4037$

\begin{abstract}
The present study aimed to investigate whether the mammalian target of rapamycin (mTOR) signaling pathway is activated in invasive breast cancer. The expression levels of phosphorylated (p)-mTOR at ser 2448 were detected, as well as the expression levels of its downstream signaling molecules: Eukaryotic translation initiation factor 4E-binding protein 1 (4E-BP1), and p70 ribosomal protein S6 kinase 1 (S6K1). The correlation between p-mTOR, p-4E-BP1, p-S6K1, and the clinicopathological parameters of breast cancer were also determined. p-mTOR, p-4E-BP1 and p-S6K1 expression was detected in 285 breast cancer tumor samples and adjacent normal tissue samples using immunohistochemistry. The expression levels and the location of the proteins were analyzed and compared in the various tissue samples. Multivariate Cox regression was used to analyze the clinicopathological factors and prognosis associated with the tissue samples. The disease-free survival rate was examined using survival analyses and Log-rank tests. The results of the present study indicated that the expression levels of p-mTOR, p-4E-BP1, and p-S6K1 were significantly higher in breast cancer tissue, as compared with normal tissue $(\mathrm{P}<0.01)$. p-mTOR was predominantly expressed in the cytoplasm, whereas p-4E-BP1 and p-S6K1 were predominantly co-expressed in the cytoplasm and the nucleus. In addition, p-4E-BP1 and p-S6K1 were more likely to be expressed in the cytoplasm in breast cancer tissue
\end{abstract}

Correspondence to: Dr Gang Sun, Department of Breast and Neck, The Affiliated Tumor Hospital of Xinjiang Medical University, 789 East Suzhou Street, Urumqi, Xinjiang 830011, P.R. China E-mail: sung853219@gmail.com

Key words: phosphorylated mammalian target of rapamycin, phosphorylated eukaryotic translation initiation factor 4E-binding protein 1, phosphorylated p70 ribosomal protein S6 kinase 1, breast cancer, prognosis samples, as compared with normal tissue samples $(\mathrm{P}<0.001)$. Positive p-mTOR was not significantly correlated with positive p-4E-BP1 and p-S6K1 expression. The survival analyses of the patients with positive p-mTOR, p-4E-BP1, and p-S6K1 tissue samples were not significantly different from those of the patients with negative tissue samples $(\mathrm{P}>0.05)$. Thus suggesting that these markers are not adequate risk factors for disease free survival $(\mathrm{P}>0.05)$. In conclusion, the results of the present study suggested that p-mTOR, p-4E-BP1, and p-S6K1 are activated in invasive breast cancer. In addition, the exclusive expression of p-4E-BP1 and p-S6K1 in the cytoplasm may be characteristic of progressive breast cancer. However, p-mTOR, p-4E-BP1, and p-S6K1 are not prognostic factors for breast cancer.

\section{Introduction}

Breast cancer is the most common cancer diagnosed in women worldwide (1). In recent years, the incidence of breast cancer has rapidly increased in economically developed regions (2). Although China has a relatively low incidence of breast cancer, the rate of diagnosis is currently increasing at an average of $3-4 \%$ per year (3). The implementation of systemic therapeutic strategies including targeted therapy, such as estrogen receptor modulators tamoxifen and raloxifene, has reduced the risk of breast cancer (4). Together with mammography screening and the improvement of localized therapy, these techniques have likely contributed to the improvements in breast cancer survival (5).

Following recent advances in cancer research, scientists have begun to attach more importance to targeted therapy. Mammalian target of rapamycin (mTOR) is a novel target that may help treat malignancies. It is a $290 \mathrm{kDa}$ molecule which functions as a serine-threonine kinase by regulating cell growth and metabolism in mTOR signaling pathway (6). The molecule has an important role in cellular processes, including apoptosis, transcription, translation, metabolism, angiogenesis, and cell cycle regulation, all of which are associated with the occurrence and development of numerous types of tumor (7-9). mTOR belongs to the family of phosphatidylinositol kinase-related 
kinases (10), and is activated by phosphorylation of ser2448 through the phosphatidylinositol 3-kinase (PI3K)/Akt signaling pathway (11). mTOR is involved in two independent signaling pathways depending on which type of complex it forms: mTORC1 or mTORC2. In the mTORC1 complex mTOR is thought to be predominantly phosphorylated at ser2448 (12). mTORC1 is involved in the P13K-Akt signaling pathway, where it mediates the activation of the eukaryotic translation initiation factor $4 \mathrm{E}$ (eIF4E) binding protein 1 (4E-BP1), and the p70 ribosomal protein S6 kinase 1 (S6K1). Together, 4E-BP1 and S6K1 control protein synthesis (13). In addition, mTOR phosphorylates S6K1 and 4E-BP1 leading to the translation of mRNA that encodes ribosomal proteins and elongation factors, as well as proteins responsible for ribosome recruitment and initiation of translation $(14,15)$. Alterations in the signaling pathway that leads to mTOR activation results in increased protein biosynthesis, cell growth, and tumor development (16). Thus suggesting that the mTORC1 signaling pathway controls protein translation and has an important role in the mechanism of tumor cell growth.

The activation of mTORC 1 and the overexpression of mTOR has been demonstrated in numerous types of cancer, including hepatocellular, renal cell, and prostate cancer (17). In addition, mutations in the phosphatidylinositol-4,5-bisphosphate 3-kinase, catalytic subunit $\alpha$ gene, phosphatase and tensin homolog (PTEN) loss, and aberrant activation of Akt have all been described in breast cancer $(18,19)$. Therefore, targeting the mTOR signaling pathway has emerged as a promising therapeutic option. Furthermore, it has been shown that mTOR activation, caused by either PTEN mutation or AKT overexpression, is notably susceptible to mTOR inhibitors $(14,20)$. Treatment of cancer with mTOR inhibitors has produced promising results in various malignancies, including prostate cancer, malignant glioma, leukemia, lymphoma, melanoma, and renal cell cancer, as well as breast cancer (21). Three mTOR antagonists: Everolimus, temsirolimus, and deforolimus (AP23573), have exhibited activity against breast cancer in preclinical studies (22-24). Various components of the mTOR signaling pathway have been recognized as attractive therapeutic targets for cancer therapy, including breast, ovarian, colon, pancreatic and gastric cancers (25-31).

The aberrant activation of the PI3K/Akt/mTOR signaling pathway is involved in oncogenesis and the progression of breast cancer (28-31). In vitro and in vivo investigations, as well as preclinical studies into drug therapies targeting the constituents of this particular signaling pathway, have been shown to exhibit significantly beneficial effects in the treatment of certain subsets of breast cancer $(20,28,32,33)$. The mechanism underlying the mTOR signaling pathway in invasive breast cancer is complex, and numerous studies have been carried out in an attempt to elucidate it (34-36). However, there has been little research conducted regarding the expression levels of phosphorylated (p)-mTOR, or the activation of its downstream signaling components p-4E-BP1 and p-S6K1 in human invasive breast cancer tissues. The aim the present study was to investigate the expression levels and location of p-mTOR and its downstream signaling pathway components, as well as their association with the clinical characteristics of breast cancer. Understanding these issues may allow mTOR to be successfully used as a therapeutic target in breast cancer treatment.

\section{Materials and methods}

Patient selection. The present study was approved by the ethics committee of the Affiliated Tumor Hospital of Xinjiang Medical University (Urumqi, China). A total of 285 patients (age, 28-73 years) with breast cancer were recruited to the present study between March 2005 and September 2009 at the Affiliated Tumor Hospital of Xinjiang Medical University. The patients provided written informed consent. The patients were followed up until March 2012. All of the patients were treated with adjuvant therapy following surgery. The clinical information of the patients was obtained from medical records, which included patient gender, age, nationality, tumor size, and lymph node metastasis, and was retrospectively reviewed for each patient. All of the patients included in the present study had invasive ductal cancer or invasive lobular cancer. No chemotherapy, endocrine therapy, or radiotherapy was performed prior to surgery. No other types of malignancy were diagnosed prior to or simultaneously to the diagnosis of breast cancer. The patients were followed-up for a median of 71.8 months, during which time a total of 60 patients suffered recurrence, metastasis, or death. The archival formalin-fixed, paraffin-embedded tumor specimens were sectioned and stained with hematoxylin and eosin (Shanghai Huayi Bio-tech Co., Ltd., Shanghai, China) in order to confirm the diagnosis under a microscope (Olympus CX4; Shanghai Yubo Biological Technology, Shanghai, China).

Immunohistochemistry. The tumor and adjacent normal breast tissue samples were $10 \%$ formalin-fixed and paraffin-embedded (Shanghai Shybio Technology). The normal breast tissue samples were used as an internal positive control. The most representative paraffin block for each tumor was selected, and $3 \mu \mathrm{m}$ tissue sections were cut from the tumor for immunohistochemical analysis. The slides containing the tissue sections were placed in a $62^{\circ} \mathrm{C}$ oven for $2 \mathrm{~h}$. The paraffin was subsequently removed and the sections were dehydrated using xylene (1 L; 2 times; 10 min; Shanghai Shybio Technology) and ethanol (1 L; 2x 100\%, $10 \mathrm{~min}$; 1x 95\%, $5 \mathrm{~min}$; 1x 80\%, $5 \mathrm{~min} ; 1 \mathrm{x} 70 \%, 5 \mathrm{~min})$. The slides were then washed three times in phosphate buffered saline (PBS) $(0.1 \mathrm{~mol} / \mathrm{ml}$; $\mathrm{pH} 7.4)$ for $5 \mathrm{~min}$. The sections were subsequently placed in sodium citrate buffer (0.01 mol/1, pH 6.0; Shanghai Shybio Technology) and boiled at $95^{\circ} \mathrm{C}$ for $20 \mathrm{~min}$, for antigen retrieval. The sections were then slowly cooled at room temperature for $\leq 20 \mathrm{~min}$, prior to being washed a further three times with PBS. The sections were then incubated for $20 \mathrm{~min}$ at room temperature, and $100 \mu 13 \% \mathrm{H}_{2} \mathrm{O}_{2}(100 \mu \mathrm{l})$ was added to inhibit endogenous peroxidase activity. Subsequently, the sections were washed with PBS and incubated with $10 \%$ newborn fetal calf serum (0.1 ml; Shanghai Shybio Technology) at room temperature for $20 \mathrm{~min}$, prior to being further washed with PBS. The primary antibodies were diluted according to the conditions listed below, and the sections were incubated overnight at $4{ }^{\circ} \mathrm{C}$. The sections were then warmed to room temperature for $1 \mathrm{~h}$ and washed with PBS. The secondary antibody was diluted according to the conditions listed below, and the sections were incubated with it for $1 \mathrm{~h}$ at room temperature, prior to being washed with PBS. The sections were then stained with $0.03 \%$ 3,3'-diaminobenzidine (Shanghai Shybio Technology) 
for $8 \mathrm{sec}$, and placed into a cylinder containing $\mathrm{dd}_{2} \mathrm{O}$ prior to being restained with $0.2 \mathrm{ml}$ hematoxylin for $30 \mathrm{sec}$, washed with water for $5 \mathrm{~min}$, placed into hydrochloric acid and ethanol $(1 \%, 100 \mathrm{ml})$ for $1 \mathrm{sec}$, and washed again for $5 \mathrm{~min}$. The sections were then dehydrated with ethanol, dried, and sealed using neutral resin (Beijing Zhongshan Golden Bridge Biological Technology Co., Ltd., Beijing, China) for microscopic examination (Olympus CX4), under which the nuclei appeared stained blue or purple and the positive products were stained brown or yellow.

For immunohistochemical staining, the sections were incubated with the following primary antibodies: Rabbit anti-human p-mTOR (Ser2448) (clone 49F9; cat. no. 2976L; Cell Signaling Technology, Inc., Danvers, MA, USA) at a dilution of 1:100; rabbit anti-human p-S6K1 (T389) (clone E175; cat. no. ab32359; Abcam, Cambridge, UK) at a dilution of 1:50, rabbit anti-human p-4E-BP1 (Thr70) [clone EPR654(2) A; cat. no. 04-1139; EMD Millipore, Billerica, MA, USA] at a dilution of 1:100, and rabbit anti-human ki-67 (clone EP5; cat. no.ZA0502; Beijing Zhongshan Golden Bridge Biological Technology Co.,Ltd.). Horseradish peroxidase-conjugated goat anti-rabbit secondary antibody (Cell Signaling Technology, Inc.) was used at a dilution of 1:100. The omission of primary antibody was used as a negative control.

Assessment of immunostaining. Assessment of immunostaining was conducted objectively by two pathologists who had yet to review the clinical data of the patients. Each pathologist independently observed and scored the immunohistochemistry results with regards to both the extent and intensity of the staining. In all discordant cases, mutual agreement was reached. The expression of p-mTOR, p-4E-BP1, and p-S6K1 was examined in both the cytoplasm and plasma membranes. Immunohistochemical staining was assessed according to the immunoreactive score (IRS), which evaluates the proportion and intensity of staining (37). Staining intensity was graded as 0 (negative), 1 (weak), 2 (moderate), or 3 (strong); the percentage of positive cells examined was scored as 0 (negative), $1(<10 \%), 2(11-50 \%), 3(51-80 \%)$, or $4(>80 \%)$. The two scores were multiplied and the IRS (values between 0 and 12) was determined: 0 , negative; $1-3$, weak; $4-6$, positive; and 8-12, strongly positive. A final score $>3$ was considered positive. The expression of Ki- 67 was considered positive if $>14 \%$ cells stained.

Statistical analysis. All statistical analyses were conducted using SPSS 17.0 (SPSS Inc., Chicago, IL, USA). A $\chi^{2}$ test was initially used in order to compare the characteristic percentages between different variables. A survival analysis and Log-rank test were carried out to compare disease free survival in the patients. Multivariate Cox regression model was subsequently built to analyze the relationship between clinicopathological factors and prognosis. $\mathrm{P}<0.05$ was considered to indicate a statistically significant difference.

\section{Results}

Expression status and cytoplasmic expression. p-mTOR, p-4E-BP1, and p-S6K1 expression was assessed using standard immunohistochemistry. Fig. 1 shows a representative immunohistochemical staining of negative and positive p-mTOR, p-4EBP1, and p-S6K1 tissue samples. The presence of brown staining of the nuclei and/or cytoplasm indicated immunoreactive tissues. The percentage of breast cancer tissue expressing p-mTOR, p-4E-BP1, and p-S6K1 was significantly higher, as compared with normal breast tissue $(\mathrm{P}=0.001, \mathrm{P}<0.001$, and $\mathrm{P}<0.001$, respectively) (Table I). In the cancerous and normal tissue samples, the positive expression of p-mTOR, p-4E-BP1, and p-S6K1 were 72 and 60\%, 26 and $8 \%$, and 42 and 18\%, respectively. Both nuclear and cytoplasmic staining was observed. As demonstrated in Table II, the location of the three phosphorylated proteins was compared between the cancerous and the normal tissue. p-mTOR was predominantly observed in the cytoplasm, and there was no significant difference between the location of mTOR in the cancerous and normal tissue $(\mathrm{P}=0.881)$, whereas both p-4E-BP1 and p-S6K1 were co-expressed in the nucleus and the cytoplasm. The exclusive cytoplasmic expression of these two phosphorylated proteins was markedly more common in cancerous tissue, as compared with normal tissue (both $\mathrm{P}<0.001$ ).

As shown in Table III, no statistical correlation was observed between the expression of p-mTOR, and p-4E-BP1 or p-S6K1 ( $\mathrm{P}=0.289$, and 0.159 , respectively), although a greater number of positive $\mathrm{p}-4 \mathrm{E}-\mathrm{BP} 1$ and $\mathrm{p}-\mathrm{S} 6 \mathrm{~K} 1$ tissue samples were found in conjunction with positive p-mTOR tissue samples. This lack of correlation may be the result of lower rates of positive p-4E-BP1 and p-S6K1, as compared with p-mTOR.

Clinicopathological significance. Table IV presents the correlation between the clinicopathological factors and p-mTOR, p-4E-BP1, and p-S6K1 expression. The results indicate that significantly reduced expression of p-mTOR is present in Ki-67 positive tissue (69, vs. 82\%, $\mathrm{P}=0.028)$. Similarly, p-S6K1 expression levels were also lower in Ki-67 positive cases (38, vs. 55\%, $\mathrm{P}=0.011)$. Positive $\mathrm{p}-\mathrm{S} 6 \mathrm{~K} 1$ expression was higher in grade I breast cancer tumors, but was similar between grade II and III tumors (88, vs. 40 and $43 \%, \mathrm{P}<0.05)$. p-4E-BP1 expression did not correlate with any of the clinicopathological factors listed $(\mathrm{P}>0.05)$, and no significance was found between the proteins and other clinocopathological factors.

Since Uyghur and Han ethnicities constitute the majority of the Xinjiang population, the expression of p-mTOR, p-4E-BP1, and p-S6K1 was analyzed according to Uyghur and Han nationality. The results presented in Table $\mathrm{V}$ exhibit the presence of statistically significant differences between both populations for: p-4E-BP1 expression in cancer (62 and 38\% respectively; $\mathrm{P}=0.020$ ), $\mathrm{p}-4 \mathrm{E}-\mathrm{BP} 1$ expression in normal tissue ( 29 and $71 \%$ respectively; $\mathrm{P}=0.029)$, and $\mathrm{p}-\mathrm{S} 6 \mathrm{~K} 1$ expression in normal tissue (32 and 68\% respectively; $\mathrm{P}=0.004$ ); however, no statistically significant differences were present between the populations for $\mathrm{p}$-mTOR expression in cancer and normal tissue $(\mathrm{P}=0.067$ vs 0.485$)$, or $\mathrm{p}-\mathrm{S} 6 \mathrm{~K} 1$ expression in cancer $(\mathrm{P}=0.695)$. These results demonstrated that the expression of p-mTOR, p-4E-BP1, p-S6K1, and Ki-67, as well as nationality, exhibited statistically significant differences between breast cancer and adjacent normal tissue samples. Therefore, clinically important factors including age, body mass index (BMI), tumor size, lymph node, stage, grade, and histological type were included in a multivariate analysis. The results of the multivariate analysis demonstrated 
Table I. Expression of p-mTOR, p-4E-BP1, and p-S6K1 in breast cancer and adjacent normal tissue samples.

\begin{tabular}{|c|c|c|c|}
\hline Expression & + & - & P-value \\
\hline \multicolumn{4}{|l|}{ p-mTOR } \\
\hline Cancer tissues & $206(72 \%)$ & $79(28 \%)$ & 0.001 \\
\hline Normal tissues & $170(60 \%)$ & $115(40 \%)$ & \\
\hline \multicolumn{4}{|l|}{ p-4E-BP1 } \\
\hline Cancer tissues & $74(26 \%)$ & $211(74 \%)$ & $<0.001$ \\
\hline Normal tissues & $24(8 \%)$ & $261(92 \%)$ & \\
\hline \multicolumn{4}{|l|}{ p-S6K1 } \\
\hline Cancer tissues & $120(42 \%)$ & $165(58 \%)$ & $<0.001$ \\
\hline Normal tissues & $50(18 \%)$ & $235(82 \%)$ & \\
\hline
\end{tabular}

p-mTOR, phosphorylated mammalian target of rapamycin; p-4E-BP1, phosphorylated eukaryotic translation initiation factor 4E binding protein; p-S6K1, phosphorylated p70 ribosomal protein S6 kinase.

Table II. Location of p-mTOR, p-4E-BP1, and p-S6K1 in breast cancer and adjacent normal tissue samples.

\begin{tabular}{lccc}
\hline Expression & Nucleus & Cytoplasm & Nucleus and cytoplasm \\
\hline p-mTOR & & & \\
Cancer tissues & $1(0 \%)$ & $270(97 \%)$ & $8(3 \%)$ \\
Normal tissues & $0(0 \%)$ & $234(98 \%)$ & $5(2 \%)$ \\
p-4E-BP1 & $3(2 \%)$ & $45(28 \%)$ & $113(70 \%)$ \\
Cancer tissues & $1(1 \%)$ & $6(7 \%)$ & $74(91 \%)$ \\
Normal tissues & $3(1 \%)$ & $28(12 \%)$ & $195(86 \%)$ \\
p-S6K1 & $6(4 \%)$ & $0(0 \%)$ & $130(96 \%)$ \\
Cancer tissues & & & $<0.001$ \\
Normal tissues & & & \\
\hline
\end{tabular}

p-mTOR, phosphorylated mammalian target of rapamycin; p-4E-BP1, phosphorylated eukaryotic translation initiation factor 4E binding protein; p-S6K1, phosphorylated p70 ribosomal protein S6 kinase.

Table III. Correlation between p-mTOR, p-4E-BP1, and p-S6K1 expression in breast cancer.

\begin{tabular}{lccc}
\hline & \multicolumn{2}{c}{ p-mTOR } & r value \\
\cline { 3 - 3 } Expression & + & - & 0.063 \\
\hline p-4E-BP1 & $57(77 \%)$ & $17(23 \%)$ & 0.289 \\
+ & $149(71 \%)$ & $62(29 \%)$ & 0.084 \\
- & & & \\
p-S6K1 & $92(77 \%)$ & $28(23 \%)$ & 0.159 \\
- & $114(69 \%)$ & $51(31 \%)$ & \\
\hline
\end{tabular}

p-mTOR: phosphorylated mammalian target of rapamycin; p-4E-BP1: phosphorylated eukaryotic translation initiation factor 4E binding protein; p-S6K1: phosphorylated p70 ribosomal protein S6 kinase.

that nationality $(\mathrm{P}<0.001), \mathrm{BMI}(\mathrm{P}=0.048), \mathrm{Ki}-67$ expression $(\mathrm{P}=0.004)$, and $\mathrm{p}-\mathrm{S} 6 \mathrm{~K} 1$ expression $(\mathrm{P}<0.001)$ were all associated with disease free survival (Table VI). The disease free survival rates were also compared by survival analysis (Fig. 2), and no statistical significance was shown between positive and negative p-mTOR, p-4E-BP1 or p-S6K1 expression $(\mathrm{P}=0.619,0.337$, and 0.704 , respectively).

\section{Discussion}

Understanding the mTOR signaling pathway is critical to the development of novel mTOR-inhibiting therapeutic strategies in the treatment of breast cancer. The majority of research conducted prior to the present study focused on breast cancer in general. The present study, however, investigated the 
Table IV. Correlation between the expression of p-mTOR, p-4E-BP1, and p-S6K1 in cancer tissue samples, and clinicopathological factors.

\begin{tabular}{|c|c|c|c|c|c|c|}
\hline \multirow[b]{2}{*}{ Characteristic } & \multicolumn{2}{|c|}{ p-mTOR } & \multicolumn{2}{|c|}{ p-4E-BP1 } & \multicolumn{2}{|c|}{ p-S6K1 } \\
\hline & + & - & + & - & + & - \\
\hline \multicolumn{7}{|l|}{ Age (years) } \\
\hline$\leq 45$ & $111(71 \%)$ & $46(29 \%)$ & $37(24 \%)$ & $120(76 \%)$ & $68(43 \%)$ & $89(57 \%)$ \\
\hline$>45$ & $95(74 \%)$ & $33(26 \%)$ & $37(29 \%)$ & $91(71 \%)$ & $52(41 \%)$ & $76(59 \%)$ \\
\hline $\mathrm{P}$-value & 0.509 & & 0.306 & & 0.648 & \\
\hline \multicolumn{7}{|l|}{ Nationality } \\
\hline Uyghur & $111(77 \%)$ & $33(23 \%)$ & $46(32 \%)$ & $98(68 \%)$ & $59(41 \%)$ & $85(59 \%)$ \\
\hline Han & $95(67 \%)$ & $46(33 \%)$ & $28(20 \%)$ & $113(80 \%)$ & $61(43 \%)$ & $80(57 \%)$ \\
\hline $\mathrm{P}$-value & 0.067 & & 0.020 & & 0.695 & \\
\hline \multicolumn{7}{|l|}{ BMI } \\
\hline$<28.0$ & $159(72 \%)$ & $61(28 \%)$ & $54(25 \%)$ & $166(75 \%)$ & $96(44 \%)$ & $124(56 \%)$ \\
\hline$\geq 28.0$ & $47(72 \%)$ & $18(28 \%)$ & $20(31 \%)$ & $45(69 \%)$ & $24(37 \%)$ & $41(63 \%)$ \\
\hline $\mathrm{P}$-value & 0.996 & & 0.315 & & 0.335 & \\
\hline \multicolumn{7}{|l|}{ Tumor size } \\
\hline $\mathrm{T} 1$ & $84(76 \%)$ & $27(24 \%)$ & $30(27 \%)$ & $81(73 \%)$ & $48(43 \%)$ & $63(57 \%)$ \\
\hline $\mathrm{T} 2, \mathrm{~T} 3, \mathrm{~T} 4$ & $122(70 \%)$ & $52(30 \%)$ & $44(25 \%)$ & $130(75 \%)$ & $72(41 \%)$ & $102(59 \%)$ \\
\hline P-value & 0.306 & & 0.744 & & 0.756 & \\
\hline \multicolumn{7}{|l|}{$\mathrm{LN}$} \\
\hline+ & $117(73 \%)$ & $44(27 \%)$ & $37(23 \%)$ & $124(77 \%)$ & $72(45 \%)$ & $89(55 \%)$ \\
\hline- & $89(72 \%)$ & $35(28 \%)$ & $37(30 \%)$ & $87(70 \%)$ & $48(39 \%)$ & $76(61 \%)$ \\
\hline $\mathrm{P}$-value & 0.867 & & 0.191 & & 0.308 & \\
\hline \multicolumn{7}{|l|}{ Stage } \\
\hline I & $47(72 \%)$ & $18(28 \%)$ & $16(25 \%)$ & $49(75 \%)$ & $27(42 \%)$ & $38(58 \%)$ \\
\hline II-IV & $159(72 \%)$ & $61(28 \%)$ & $58(26 \%)$ & $162(74 \%)$ & $93(42 \%)$ & $127(58 \%)$ \\
\hline $\mathrm{P}$-value & 0.996 & & 0.778 & & 0.916 & \\
\hline \multicolumn{7}{|l|}{ Tumor grade } \\
\hline I & $5(63 \%)$ & $3(38 \%)$ & $3(38 \%)$ & $5(63 \%)$ & $7(88 \%)$ & $1(13 \%)$ \\
\hline II & $147(72 \%)$ & $58(28 \%)$ & $52(25 \%)$ & $153(75 \%)$ & $82(40 \%)$ & $123(60 \%)$ \\
\hline III & $54(75 \%)$ & $18(25 \%)$ & $19(26 \%)$ & $53(74 \%)$ & $31(43 \%)$ & $41(57 \%)$ \\
\hline P-value & 0.717 & & 0.757 & & 0.029 & \\
\hline \multicolumn{7}{|l|}{ Histological type } \\
\hline IDC & $177(73 \%)$ & $67(27 \%)$ & $68(28 \%)$ & $176(72 \%)$ & $105(43 \%)$ & $139(57 \%)$ \\
\hline IDC+ILC & $21(70 \%)$ & $9(30 \%)$ & $5(17 \%)$ & $25(83 \%)$ & $11(37 \%)$ & $19(63 \%)$ \\
\hline ILC & $8(73 \%)$ & $3(27 \%)$ & $1(9 \%)$ & $10(91 \%)$ & $4(36 \%)$ & $7(64 \%)$ \\
\hline P-value & 0.958 & & 0.139 & & 0.739 & \\
\hline \multicolumn{7}{|l|}{$\mathrm{Ki}-67$} \\
\hline+ & $146(69 \%)$ & $66(31 \%)$ & $60(28 \%)$ & $152(72 \%)$ & $80(38 \%)$ & $132(62 \%)$ \\
\hline- & $60(82 \%)$ & $13(18 \%)$ & $14(19 \%)$ & $59(81 \%)$ & $40(55 \%)$ & $33(45 \%)$ \\
\hline P-value & 0.028 & & 0.125 & & 0.011 & \\
\hline \multicolumn{7}{|c|}{ Recurrence, metastasis and death } \\
\hline+ & $41(68 \%)$ & $19(32 \%)$ & $12(20 \%)$ & $48(80 \%)$ & $25(42 \%)$ & $35(58 \%)$ \\
\hline- & $165(73 \%)$ & $60(27 \%)$ & $62(28 \%)$ & $163(72 \%)$ & $95(42 \%)$ & $130(58 \%)$ \\
\hline $\mathrm{P}$-value & 0.442 & & 0.236 & & 0.938 & \\
\hline
\end{tabular}

p-mTOR, phosphorylated mammalian target of rapamycin; p-4E-BP1, phosphorylated eukaryotic translation initiation factor 4E binding protein; p-S6K1, phosphorylated p70 ribosomal protein S6 kinase; BMI, body mass index; LN, lymph node; IDC, invasive ductal carcinoma; ILC, invasive lobular carcinoma.

expression levels of p-mTOR, as well as its phosphorylated downstream signaling components S6K1 and 4E-BP1, in invasive ductal and lobular breast cancer. The results of the present study indicated that patients with breast cancer may benefit from treatment with mTOR inhibitors. since the majority of the breast cancer tissue samples (72\%) exhibited positive immunostaining for $\mathrm{p}-\mathrm{mTOR}$.

The results from the investigation into $\mathrm{p}$-mTOR expression are concordant with those of previous studies. Bose et al (30) showed that p-mTOR was expressed in $82 \%$ of invasive breast 
Table V. Comparison of the expression of p-mTOR, p-4E-BP1, and $\mathrm{p}-\mathrm{S} 6 \mathrm{~K} 1$ between various nationalities.

\begin{tabular}{lccc}
\hline Expression & Uyghur & Han & P-value \\
\hline $\begin{array}{l}\text { p-mTOR in cancer } \\
+\end{array}$ & $111(54 \%)$ & $95(46 \%)$ & 0.067 \\
- & $33(42 \%)$ & $46(58 \%)$ & \\
p-mTOR in normal tissue & & & \\
+ & $83(49 \%)$ & $87(51 \%)$ & 0.485 \\
- & $61(53 \%)$ & $54(47 \%)$ & \\
p-4E-BP1 in cancer & & & \\
+ & $46(62 \%)$ & $28(38 \%)$ & 0.020 \\
- & $98(46 \%)$ & $113(54 \%)$ & \\
p-4E-BP1 in normal tissue & & & \\
+ & $7(29 \%)$ & $17(71 \%)$ & 0.029 \\
- & $137(52 \%)$ & $124(48 \%)$ & \\
p-S6K1 in cancer & & & \\
+ & $59(49 \%)$ & $61(51 \%)$ & 0.695 \\
- & $85(52 \%)$ & $80(48 \%)$ & \\
p-S6K1 in normal tissue & & & \\
+ & $16(32 \%)$ & $34(68 \%)$ & 0.004 \\
- & $128(54 \%)$ & $107(46 \%)$ & \\
\hline
\end{tabular}

p-mTOR, phosphorylated mammalian target of rapamycin; p-4E-BP1, phosphorylated eukaryotic translation initiation factor $4 \mathrm{E}$ binding protein; p-S6K1, phosphorylated p70 ribosomal protein S6 kinase.

cancer cases. In addition, in a triple negative breast cancer (TNBC) study, the majority of tumors $(72.1 \%)$ were shown to be p-mTOR positive (38). The present study also demonstrated that the phosphorylated, and therefore active, downstream molecules 4E-BP1 and S6K1 were both highly expressed in breast cancer. Upon phosphorylation by mTOR, the binding protein and translational repressor 4E-BP1 releases the initiation factor eIF4E. Phosphorylation of 4E-BP1 disrupts its interaction with the eIF4E translation initiation factor, enabling eIF4E to bind to the cap structure at the 5' end of mRNAs, which subsequently promotes ribosomal recruitment and initiation of translation (15). Rojo et al (39) demonstrated that p-4EBP1 was the main mTOR signaling pathway factor associated with the prognosis and grade of malignancy in breast tumors. Furthermore, p-4EBP1 was detected in both human epidermal growth factor (HER2)-positive and HER2-negative tumors.

mTOR phosphorylates p70S6K, which in turn leads to the phosphorylation of ribosomal protein S6, and subsequently to the translation of mRNA that encodes ribosomal proteins and elongation factors (40). In a tissue-array-based analysis, p-S6 expression was detected in 83\% (116) of ductal carcinoma in situ (DCIS) and 94\% (137) of invasive cancer samples, higher than the results of the present study. A statistically significant difference was reported between the percentage of DCIS cases overexpressing p-S6 (47\%; 54-116), and the percentage of invasive cancers overexpressing p-S6 (72\%; 99-137) (30).

mTOR is thought to be located predominantly in the cytoplasm and membrane fractions of cells. Little research has been conducted regarding whether the activated (phosphorylated) form of mTOR is also located in the same fractions.
Table VI. Disease-free survival multivariate analyses.

\begin{tabular}{lccc}
\hline Characteristic & HR & $95 \%$ CI & P-value \\
\hline Age & 1.210 & $0.916-1.599$ & 0.179 \\
Nationality & 0.104 & $0.071-0.155$ & $<0.001$ \\
BMI & 0.707 & $0.502-0.997$ & 0.048 \\
Tumor size & 0.845 & $0.554-1.289$ & 0.434 \\
LN & 1.192 & $0.817-1.740$ & 0.362 \\
Stage & 1.043 & $0.591-0.842$ & 0.884 \\
Tumor grade & 0.872 & $0.652-1.167$ & 0.357 \\
Histological type & 0.934 & $0.672-1.299$ & 0.686 \\
Ki-67 & 0.577 & $0.398-0.836$ & 0.004 \\
p-mTOR & 0.787 & $0.571-1.085$ & 0.143 \\
p-4E-BP1 & 0.978 & $0.694-1.378$ & 0.898 \\
p-S6K1 & 2.029 & $1.431-2.876$ & $<0.001$ \\
\hline
\end{tabular}

p-mTOR, phosphorylated mammalian target of rapamycin; p-4E-BP1, phosphorylated eukaryotic translation initiation factor $4 \mathrm{E}$ binding protein; p-S6K1, phosphorylated p70 ribosomal protein S6 kinase; BMI, body mass index; LN, lymph node; HR, hazard ratio; CI, confidence interval.

Although the mTOR protein can shuttle between the nucleus and cytoplasm $(39,41)$, in the present study p-mTOR was predominantly located in the cytoplasm. This phenomenon may be due to the use of various cell types and antibodies. Korkolopoulou et al (42) previously reported that mTOR was predominantly detected in the cytoplasm-cell membrane, but was also occasionally detected in the nucleus, suggesting that the nuclear translocation of mTOR may have an important role in activating its cytoplasmic signaling. Zhang et al (43) showed that in human malignant cell lines, human fibroblasts, and murine myoblasts, mTOR is predominantly nuclear, but that in HEK293 human embryonic kidney cells mTOR is largely excluded from the nucleus. In HEK293 and Rh30 rhabdomyosarcoma cell hybrids that co-express markers unique to HEK293 (E1A) and Rh30 (MyoD), mTOR distribution was predominantly nuclear with detectable levels in the cytoplasm.

In the present study, both p-4E-BP1 and p-S6K1 were predominantly co-expressed in the nucleus and cytoplasm, and exclusive cytoplasm expression was significantly higher in cancerous tissue as compared with normal tissue. A previous study detected 4E-BP1 in cytoplasmic fractions in all cell lines, and nuclear localization of 4E-BP1 was confirmed in numerous cell lines and tissues; however, the mechanism underlying 4E-BP entry into the nucleus remains to be elucidated (44). p70S6K is a nuclear cytoplasmic shuttling protein that is activated in the nucleus by mTOR, and relocalized to the cytoplasm following the initiation of mRNA translation (45). Therefore, it is not surprising that p-S6K1 may be located in the cytoplasm and/or nucleus $(42,46)$. In addition, the downregulation of nuclear $\mathrm{p}-\mathrm{S} 6 \mathrm{~K} 1$ has been reported to be involved in the growth, invasion, and metastasis of gastric cancer, and may be used to indicate the biological behaviors of gastric cancer in clinicopathological practice (47).

Although in the present study the majority of the samples that were positive for $\mathrm{p}-4 \mathrm{E}-\mathrm{BP} 1$ and $\mathrm{p}-\mathrm{S} 6 \mathrm{~K} 1$ expression were also positive for $\mathrm{p}$-mTOR, positive $\mathrm{p}$-mTOR was not significantly correlated with positive $\mathrm{p}-4 \mathrm{E}-\mathrm{BP} 1$ and $\mathrm{p}-\mathrm{S} 6 \mathrm{~K} 1$. 

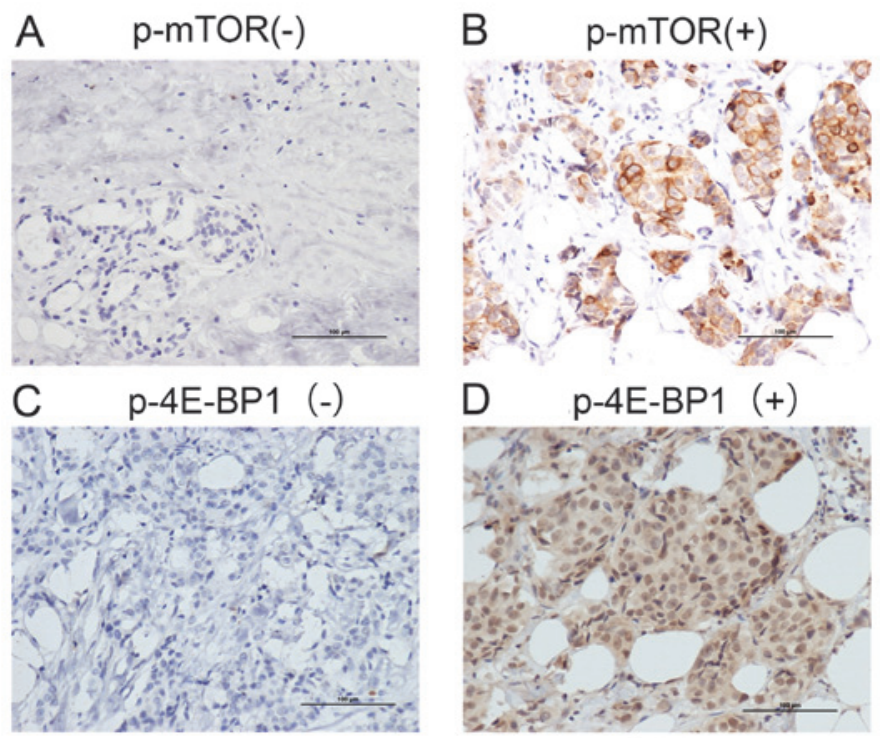

\section{D $\quad \mathrm{p}-4 \mathrm{E}-\mathrm{BP} 1(+)$}
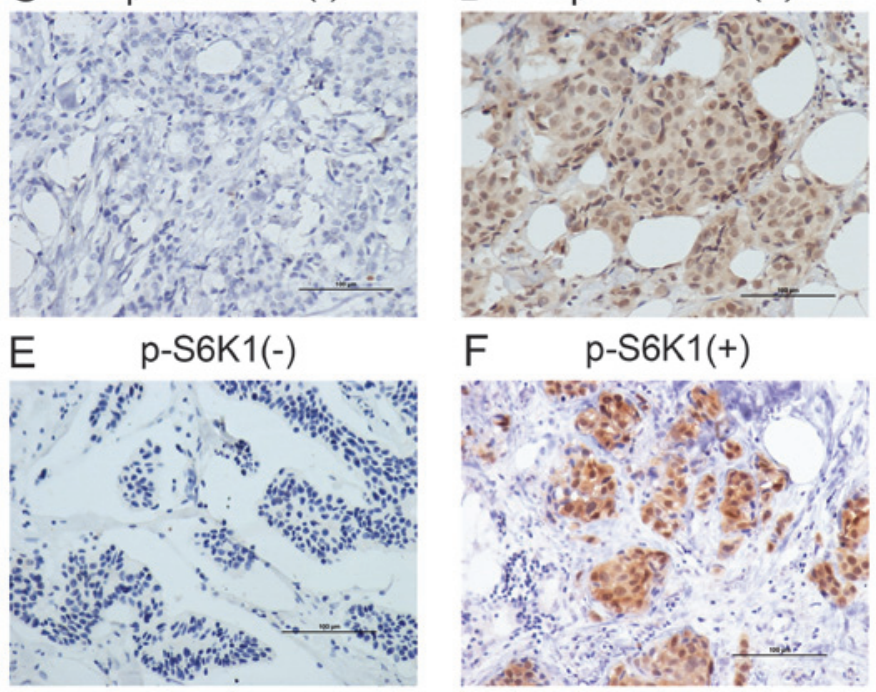

Figure 1. Immunohistochemistry of the breast cancer tumor samples. Typical tumor samples were stained with 3,3'-diaminobenzidine to stain the nuclei. (A and B) phosphorylated mammalian target of rapamycin (p-mTOR), (C and D) phosphorylated eukaryotic translation initiation factor 4E-binding protein 1 (p-4E-BP1), and (E) and (F) phosphorylated p70 ribosomal protein S6 kinase 1 (p-S6K1) expression was determined. Scale bars, $100 \mu \mathrm{m}$.
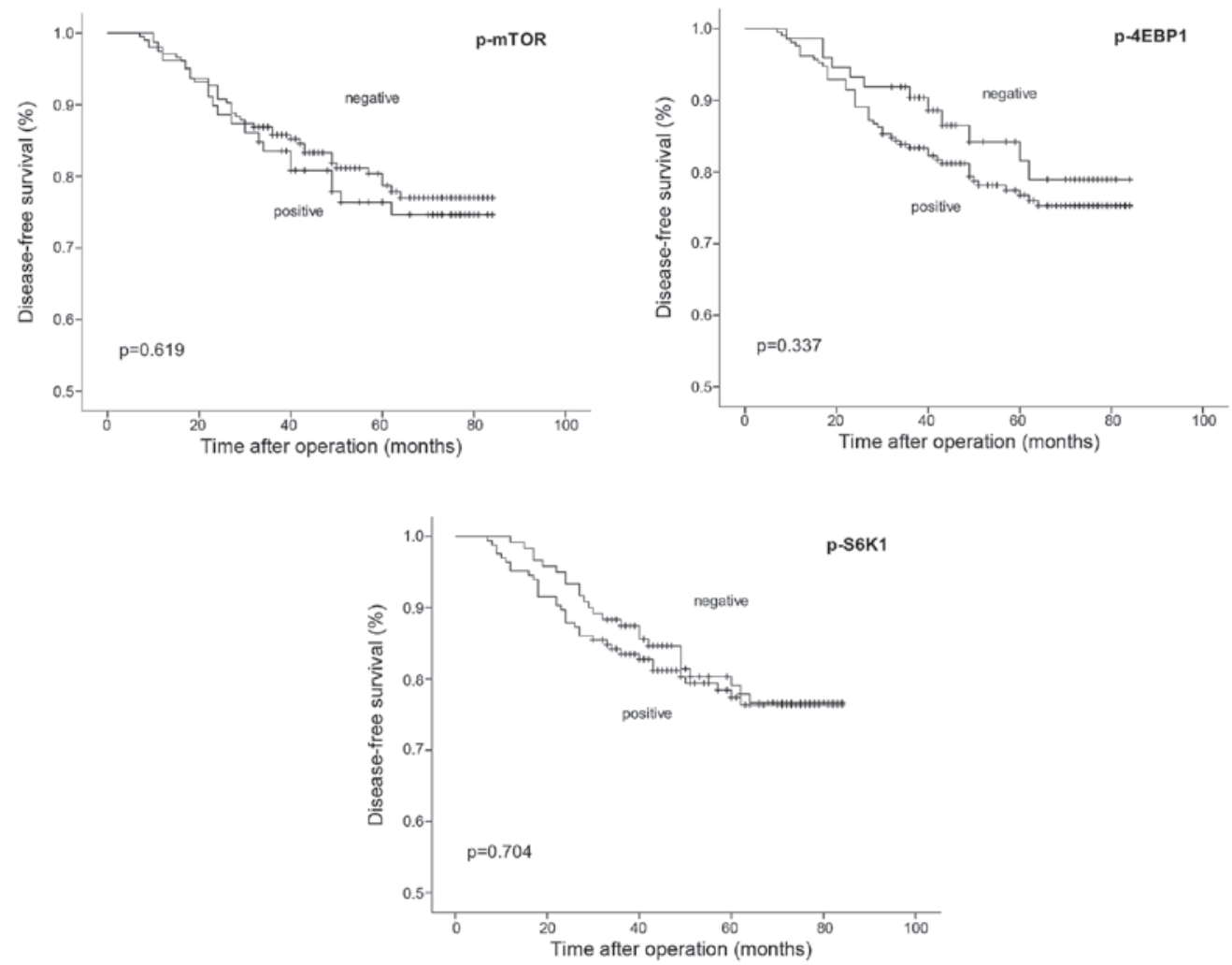

Figure 2. Survival analysis on the expression of phosphorylated mammalian target of rapamycin (p-mTOR), phosphorylated eukaryotic translation initiation factor 4E-binding protein 1 (p-4E-BP1), and phosphorylated p70 ribosomal protein S6 kinase 1 (p-S6K1). 
This may be the result of statistical differences, due to the lower rates of positive $\mathrm{p}-4 \mathrm{E}-\mathrm{BP} 1$ and $\mathrm{p}-\mathrm{S} 6 \mathrm{~K} 1$ expression. It is well established that mTOR-dependent phosphorylation of S6K1 is not linearly associated with to 4E-BP1 phosphorylation $(48,49)$. The discrepancy between p-4E-BP1 and p-S6K1 expression and $\mathrm{p}-\mathrm{mTOR}$ expression that was observed in breast cancer may be an indication that p-4E-BP1 and p-S6K1 are phosphorylated by other signaling pathways (50), and that the three proteins are topographically distinct.

Numerous studies $(31,38,39,51-53)$ have reported the clinicopathological and prognostic significance of p-mTOR, p-4E-BP1, and $\mathrm{p}-\mathrm{S} 6 \mathrm{~K} 1$, and positive correlations between the presence of the proteins and negative prognosis have been reported. In the present study however, no statistically significant correlation was demonstrated between p-mTOR, p-4E-BP1, and p-S6K1 expression and clinicopathologic variables.

A study on familial and sporadic invasive breast cancer showed that the expression of p-mTOR was correlated with lymph node status, and also had an overall negative impact on patient survival (31). Another study demonstrated that breast cancers exhibiting p-mTOR overexpression had a three times greater risk of disease recurrence (30). In addition, p-mTOR expression has been shown to be closely associated with poor prognosis in patients with cervical adenocarcinoma or hepatocellular cancer $(54,55)$. Zhou et al $(56)$ demonstrated that high p-mTOR expression levels were associated with a poor disease free survival rate in 165 patients with breast cancer; the results of this study also showed that p-mTOR was positively correlated with HER2 overexpression. Bakarakos et al (31) detected p-mTOR expression by immunohistochemistry and imaging analysis in $44.2 \%$ of 215 invasive breast cancer samples, and demonstrated that p-mTOR was positively correlated with lymph node status $(\mathrm{P}=0.010)$, and had a negative impact on survival $(\mathrm{P}=0.016)$. It appears that activation of the mTOR signaling pathway is associated with a more aggressive phenotype in both TNBC and non-TNBC breast cancers. However, few reports have investigated the effects of p-mTOR on patient survival. Annovazzi et al (53) demonstrated that no statistically significant correlation existed between p-mTOR and patient survival. In addition, Bose et al demonstrated the lack of a statistically significant correlation between p-mTOR and tumor characteristics (30). In a TNBC study, the majority of tumors (72.1\%) were p-mTOR positive; however p-mTOR expression did not correlate with age, tumor size, grade, lymph node status, or tumor stage (38).

Pelloski et al (57) demonstrated by univariate, but not multivariate, analysis that p-S6K1 was associated with reduced overall survival time. In addition, a previous study (58) showed that the p-S6K1 marker was significantly correlated with survival, using both analysis types. This may be due to the fact that the percentage of p-S6K1-negative cases in the former study $(6 \%)$ was lower than that of the latter study (44\%), or due to the fact that Pelloski et al (57) included additional markers (namely, YKL-40 and phosphorylated-extracellular-regulated kinases) in their study, which exhibited more dominant molecular effects within the tumor cells. Furthermore, Korkolopoulou et al (42) did not report any association between p-S6K1 and patient survival, and the results of this study indicated that the immunostaining of $\mathrm{p}-4 \mathrm{E}-\mathrm{BP} 1$ was nuclear. Although the underlying mechanisms of $\mathrm{p}-4 \mathrm{E}-\mathrm{BP} 1$ nuclear localization have yet to be clarified, there is evidence that eIF4E functions as a nuclear export regulator for numerous RNAs implicated in proliferation and cell growth (52). Korkolopoulou et al (42) also showed that p-4E-BP1 expression levels increased with tumor grade, whereas Ermoian et al (59) showed no correlation between the mRNA and protein expression levels of p-4E-BP1 (measured using immunoblotting) and glioma grade. p-4E-BP1 was shown to be the main signaling pathway factor associated with the prognosis and malignancy grade of breast tumors. Expression of p-4E-BP1 was correlated with a high tumor proliferation rate (39).

In conclusion, the present study used immunohistochemistry to determine the activated components of the mTORC1 signaling pathway. The results indicated that mTOR and its downstream signaling components were activated in invasive breast cancer. The location of these signaling components was not exclusive to the cytoplasm or nucleus; however, exclusive expression of p-4E-BP1 and p-S6K1 in the cytoplasm may be one of the characteristics of progressive breast cancer. p-mTOR, p-4E-BP1, and p-S6K1 did not show any statistically significant correlation with prognosis, the reasons for which require further investigation.

\section{Acknowledgements}

The authors of the present study are thankful for the pathological advice of Dr Mei Zhou, and for the statistical technical assistance provided by Assistant Professor Xuemei Yao. Financial support was provided by a grant from the Natural Science Foundation of China (grant no. 30960376).

\section{References}

1. Jemal A, Bray F, Center MM, Ferlay J, Ward E and Forman D: Global cancer statistics. CA Cancer J Clin 61: 69-90, 2011.

2. Héry C, Ferlay J, Boniol M and Autier P: Changes in breast cancer incidence and mortality in middle-aged and elderly women in 28 countries with Caucasian majority populations. Ann Oncol 19: 1009-1018, 2008.

3. Parkin DM, Bray F, Ferlay J and Pisani P: Global cancer statistics, 2002. CA Cancer J Clin 55: 74-108, 2005.

4. den Hollander P, Savage MI and Brown PH: Targeted therapy for breast cancer prevention. Front Oncol 3: 250, 2013.

5. Levi F, Bosetti C, Lucchini F, Negri E and La Vecchia C: Monitoring the decrease in breast cancer mortality in Europe. Eur J Cancer Prev 14: 497-502, 2005.

6. Guertin DA and Sabatini DM: Defining the role of mTOR in cancer. Cancer Cell 12: 9-22, 2007.

7. Dai DL, Martinka M and Li G: Prognostic significance of activated Akt expression in melanoma: A clinicopathologic study of 292 cases. J Clin Oncol 23: 1473-1482, 2005.

8. Kreisberg JI, Malik SN, Prihoda TJ, et al: Phosphorylation of Akt (Ser473) is an excellent predictor of poor clinical outcome in prostate cancer. Cancer Res 64: 5232-5236, 2004.

9. Nakanishi K, Sakamoto M, Yamasaki S, Todo S and Hirohashi S: Akt phosphorylation is a risk factor for early disease recurrence and poor prognosis in hepatocellular carcinoma. Cancer 103: 307-312, 2005.

10. Cutler NS, Heitman J and Cardenas ME: TOR kinase homologs function in a signal transduction pathway that is conserved from yeast to mammals. Mol Cell Endocrinol 155: 135-142, 1999.

11. Shaw RJ and Cantley LC: Ras, PI(3)K and mTOR signalling controls tumour cell growth. Nature 441: 424-430, 2006.

12. Copp J, Manning G and Hunter T: TORC-specific phosphorylation of mammalian target of rapamycin (mTOR): Phospho-Ser2481 is a marker for intact mTOR signaling complex 2. Cancer Res 69: 1821-1827, 2009.

13. Ma XM and Blenis J: Molecular mechanisms of mTOR-mediated translational control. Nat Rev Mol Cell Biol 10: 307-318, 2009. 
14. Proud CG: Role of mTOR signalling in the control of translation initiation and elongation by nutrients. Curr Top Microbiol Immunol 279: 215-244, 2004.

15. Gera JF, Mellinghoff IK, Shi Y, et al: AKT activity determines sensitivity to mammalian target of rapamycin (mTOR) inhibitors by regulating cyclin D1 and c-myc expression. J Biol Chem 279: 2737-2746, 2004

16. Seeliger H, Guba M, Kleespies A, Jauch KW and Bruns CJ: Role of mTOR in solid tumor systems: A therapeutical target against primary tumor growth, metastases, and angiogenesis. Cancer Metastasis Rev 26: 611-621, 2007.

17. Yu G, Wang J, Chen Y, et al: Overexpression of phosphorylated mammalian target of rapamycin predicts lymph node metastasis and prognosis of chinese patients with gastric cancer. Clin Cancer Res 15: 1821-1829, 2009.

18. Samuels Y, Wang Z, Bardelli A, et al: High frequency of mutations of the PIK3CA gene in human cancers. Science 304: 554, 2004.

19. Pérez-Tenorio G, Alkhori L, Olsson B, et al: PIK3CA mutations and PTEN loss correlate with similar prognostic factors and are not mutually exclusive in breast cancer. Clin Cancer Res 13: 3577-3584, 2007.

20. Noh WC, Mondesire WH, Peng J, et al: Determinants of rapamycin sensitivity in breast cancer cells. Clin Cancer Res 10: 1013-1023, 2004.

21. No JH, Jeon YT, Park IA, et al: Expression of mTOR protein and its clinical significance in endometrial cancer. Med Sci Monit 15: BR301-BR305, 2009.

22. Meric-Bernstam F and Gonzalez-Angulo AM: Targeting the mTOR signaling network for cancer therapy. J Clin Oncol 27: 2278-2287, 2009 .

23. Lane HA, Wood JM, McSheehy PM, et al: mTOR inhibitor RAD001 (everolimus) has antiangiogenic/vascular properties distinct from a VEGFR tyrosine kinase inhibitor. Clin Cancer Res 15: 1612-1622, 2009.

24. Yu K, Toral-Barza L, Discafani C, et al: mTOR, a novel target in breast cancer: The effect of CCI-779, an mTOR inhibitor, in preclinical models of breast cancer. Endocr Relat Cancer 8: 249-258, 2001

25. Chan S: Targeting the mammalian target of rapamycin (mTOR): A new approach to treating cancer. Br J Cancer 91: 1420-1424, 2004.

26. Georgakis GV and Younes A: From Rapa Nui to rapamycin: Targeting PI3K/Akt/mTOR for cancer therapy. Expert Rev Anticancer Ther 6: 131-140, 2006.

27. O'Reilly KE, Rojo F, She QB, et al: mTOR inhibition induces upstream receptor tyrosine kinase signaling and activates Akt. Cancer Res 66: 1500-1508, 2006.

28. Heinonen H, Nieminen A, Saarela M, et al: Deciphering downstream gene targets of $\mathrm{PI} 3 \mathrm{~K} / \mathrm{mTOR} / \mathrm{p} 70 \mathrm{~S} 6 \mathrm{~K}$ pathway in breast cancer. BMC Genomics 9: 348, 2008.

29. Dillon RL, White DE and Muller WJ: The phosphatidyl inosito 3-kinase signaling network: Implications for human breast cancer. Oncogene 26: 1338-1345, 2007.

30. Bose S, Chandran S, Mirocha JM and Bose N: The Akt pathway in human breast cancer: A tissue-array-based analysis. Mod Pathol 19: 238-245, 2006.

31. Bakarakos P, Theohari I, Nomikos A, et al: Immunohistochemical study of PTEN and phosphorylated mTOR proteins in familial and sporadic invasive breast carcinomas. Histo-pathology 56: 8 $76-88,2010$.

32. Mondesire WH, Jian W, Zhang H, et al: Targeting mammalian target of rapamycin synergistically enhances chemotherapy-induced cytotoxicity in breast cancer cells. Clin Cancer Res 10: 7031-7042, 2004.

33. Zeng Q, Yang Z, Gao YJ, et al: Treating triple-negative breast cancer by a combination of rapamycin and cyclophosphamide: An in vivo bioluminescence imaging study. Eur J Cancer 46: 1132-1143, 2010

34. Volinsky N, McCarthy CJ, von Kriegsheim A, et al: Signalling mechanisms regulating phenotypic changes in breast cancer cells. Biosci Rep 35: e00178, 2015.

35. Li G, Shan C, Liu L, et al: Tanshinone IIA inhibits HIF-1 $\alpha$ and VEGF expression in breast cancer cells via mTOR/p70S6K/ RPS6/4E-BP1 signaling pathway. PLoS One 10: e0117440, 2015.

36. Wang L, Wu J, Lu J, Ma R, Sun D and Tang J: Regulation of the cell cycle and PI3K/Akt/mTOR signaling pathway by tanshinone I in human breast cancer cell lines. Mol Med Rep 11: 931-939, 2015.
37. Remmele W and Stegner HE: Recommendation for uniform definition of an immunoreactive score (IRS) for immunohistochemical estrogen receptor detection (ER-ICA) in breast cancer tissue. Pathologe 8: 138-140, 1987 (In German).

38. Ueng SH, Chen SC, Chang YS, et al: Phosphorylated mTOR expression correlates with poor outcome in early-stage triple negative breast carcinomas. Int J Clin Exp Pathol 5: 806-813, 2012.

39. Rojo F, Najera L, Lirola J, et al: 4E-binding protein 1, a cell signaling hallmark in breast cancer that correlates with pathologic grade and prognosis. Clin Cancer Res 13: 81-89, 2007.

40. Jacinto $\mathrm{E}$ and Hall MN: Tor signalling in bugs, brain and brawn Nat Rev Mol Cell Biol 4: 117-126, 2003.

41. Bachmann RA, Kim JH, Wu AL, Park IH and Chen J: A nuclear transport signal in mammalian target of rapamycin is critical for its cytoplasmic signaling to S6 kinase 1. J Biol Chem 281: 7357-7363, 2006.

42. Korkolopoulou P, Levidou G, El-Habr EA, et al: Phosphorylated 4E-binding protein 1 (p-4E-BP1): A novel prognostic marker in human astrocytomas. Histopathology 61: 293-305, 2012.

43. Zhang X, Shu L, Hosoi H, Murti KG and Houghton PJ: Predominant nuclear localization of mammalian target of rapamycin in normal and malignant cells in culture. J Biol Chem 277: 28127-28134, 2002.

44. Rong L, Livingstone M, Sukarieh R, et al: Control of eIF4E cellular localization by eIF4E-binding proteins, 4E-BPs. RNA 14: 1318-1327, 2008.

45. Vega F, Medeiros LJ,Leventaki V,et al: Activation of mammalian target of rapamycin signaling pathway contributes to tumor cell survival in anaplastic lymphoma kinase-positive anaplastic large cell lymphoma. Cancer Res 66: 6589-6597, 2006.

46. Li XY, Zhang LQ, Zhang XG, et al: Association between $\mathrm{AKT} / \mathrm{mTOR}$ signalling pathway and malignancy grade of human gliomas. J Neurooncol 103: 453-458, 2011.

47. Xiao L, Wang YC, Li WS and Du Y: The role of mTOR and phospho-p70S6K in pathogenesis and progression of gastric carcinomas: An immunohistochemical study on tissue microarray. J Exp Clin Cancer Res 28: 152, 2009.

48. Hara K, Yonezawa K, Weng QP, Kozlowski MT, Belham C and Avruch J: Amino acid sufficiency and mTOR regulate p70 S6 kinase and eIF-4E BP1 through a common effector mechanism. J Biol Chem 273: 14484-14494, 1998.

49. Weng QP, Kozlowski M, Belham C, Zhang A, Comb MJ and Avruch J: Regulation of the p70 S6 kinase by phosphorylation in vivo. Analysis using site-specific anti-phosphopeptide antibodies. J Biol Chem 273: 16621-16629, 1998.

50. Sarbassov DD, Guertin DA, Ali SM and Sabatini DM: Phosphorylation and regulation of Akt/PKB by the rictor-mTOR complex. Science 307: 1098-1101, 2005.

51. Chaux A, Albadine R, Schultz L, et al: Dysregulation of the mammalian target of rapamycin pathway in chromophobe renal cell carcinomas. Hum Pathol 44: 2323-2330, 2013.

52. Darb-Esfahani S, Faggad A, Noske A, et al: Phospho-mTOR and phospho-4EBP1 in endometrial adenocarcinoma: Association with stage and grade in vivo and link with response to rapamycin treatment in vitro. J Cancer Res Clin Oncol 135: 933-941, 2009.

53. Annovazzi L, Mellai M, Caldera V, Valente G, Tessitore L and Schiffer D: mTOR, S6 and AKT expression in relation to proliferation and apoptosis/autophagy in glioma. Anticancer Res 29: 3087-3094, 2009.

54. van derHage JA, vanden BroekLJ,Legrand C, etal:Overexpression of P70 S6 kinase protein is associated with increased risk of locoregional recurrence in node-negative premenopausal early breast cancer patients. Br J Cancer 90: 1543-1550, 2004.

55. Zhou L, Huang Y, Li J and Wang Z: The mTOR pathway is associated with the poor prognosis of human hepatocellular carcinoma. Med Oncol 27: 255-261, 2010.

56. Zhou X, Tan M, Stone Hawthorne V, et al: Activation of the $\mathrm{Akt} / \mathrm{mammalian}$ target of rapamycin/4E-BP1 pathway by ErbB2 overexpression predicts tumor progression in breast cancers. Clin Cancer Res 10: 6779-6788, 2004

57. Pelloski CE, Lin E, Zhang L, et al: Prognostic associations of activated mitogen-activated protein kinase and Akt pathways in glioblastoma. Clin Cancer Res 12: 3935-3941, 2006.

58. Chakravarti A, Zhai G, Suzuki Y, et al: The prognostic significance of phosphatidylinositol 3-kinase pathway activation in human gliomas. J Clin Oncol 22: 1926-1933, 2004.

59. Ermoian RP, Kaprealian T,Lamborn KR, et al: Signal transduction molecules in gliomas of all grades. J Neurooncol 91: 19-26, 2009. 